\title{
Why Modeling Cultural Evolution Is Still Such a Challenge
}

\author{
Dan Sperber and Nicolas Claidière \\ Institut Jean-Nicod (CNRS, EHESS, ENS), Paris, France \\ Correspondence to: dan@sperber.com
}

The idea that cultural evolution exhibits variation, competition, and inheritance and therefore can be studied by adjusting the Darwinian theory of evolution by natural selection is an attractive one. It has been argued by a number of authors (e.g., Campbell 1960; Monod 1970; Dawkins 1976; Cavalli-Sforza and Feldman 1981; Boyd and Richerson 1985; Durham 1991; Aunger 2002; Mesoudi et al. 2004) and pursued in a variety of ways, some (Dawkins and memeticists) staying close to the Darwinian model, others (e.g., Boyd, Richerson, and their collaborators) being more innovative. We agree that there are relevant analogies between biological and cultural evolution and, in particular, that cultural items do exhibit variation, competition, and cumulative modification. On the other hand, we believe that a proper understanding of the mechanisms of cultural propagation drawing on the work of cognitive and social scientists (see Sperber and Hirschfeld 1999 for a review) contradicts the idea that culture exhibits inheritance in the strict sense needed for the theory of evolution by natural selection to apply straightforwardly to it. If so, it will take more than adjusting the Darwinian model to be faithful to the Darwinian inspiration.

\section{Peculiarities of Cultural Propagation}

Whereas the mechanisms of biological inheritance operate at a molecular level, invisible to the naked eye, and their discovery, from Mendel to Crick and Watson and beyond, has taken the form of a series of major scientific advances, the mechanisms of cultural inheritance are, to a significant extent, accessible to ordinary observation. Cultural propagation-learning, teaching, sharing of attitudes and values, and so on-takes place through the ordinary channels of information transmission, and in particular through imitation and communication. Imitation, communication, and also memory (without which cultural information would not survive to propagate) have been studied in depth by neuroscientists, psychologists, linguists, anthropologists, and sociologists. Drawing on their work, one can state with confidence that cultural propagation differs from biological propagation in crucial respects.

Among the various mechanisms involved in biological heritability (cytoplasmic heritability, sex linkage disequilibrium, epigenetic transmission, niche construction, and so on), replication of DNA is the most important and central one because it allows the faithful conservation of genetic information, and therefore the propagation of successful genotypes. Other mechanisms involved in biological inheritance play a role by contributing to this central phenomenon. Cultural propagation, on the other hand, is achieved through many different and independent mechanisms, none of which is central and none of which is a robust replication mechanism.

\section{Limits of Imitation}

Imitation is often presented as the main mechanism of cultural propagation, but, in fact, the notion is stretched to cover a wide variety of quite different processes. It might seem reasonable, for instance, to suggest that both the sounds of words and their written shapes are culturally transmitted through imitation. In fact, learning the sounds of words is a universal, automatic, highly canalized process taking place in early childhood and needing no explicit teaching. Learning the shapes of written words, on the other hand, is specific to certain cultural groups, takes place in later childhood or in adulthood and involves deliberate effort on the part of learners and teachers. Speech sounds and writing are cultural items that involve different cognitive and social mechanisms, and also different brain areas (Mazoyer et al. 1993; Cohen and Dehaene 2004). As for the meaning of words, it cannot be perceived and therefore cannot be imitated. Its cultural transmission involves yet other cognitive and social mechanisms (Bloom 2000).

\section{The Argument from Macrostability}

Still, the diversity of mechanisms involved in cultural propagation would not matter too much to the Darwinian program 
if, on the whole, these mechanisms produced the kind of heritability that makes selection possible. Darwin had good commonsense reasons to believe in the robustness of biological inheritance and this allowed him to develop his theory without any knowledge of the mechanisms that secured inheritance. Is not the reality of cultural inheritance as blatant as that of biological inheritance? Is not the very existence of culture- that is, of relatively stable representations, practices, and artifacts distributed across generations throughout a social group-sufficient evidence of the heritability of cultural items? If the microprocesses of cultural propagation failed to exhibit sufficient fidelity, how could the relative stability evidenced by the very existence of culture ever be arrived at? This argument from observed macrostability to inferred microheritability ignores the possibility that microheritability may not be the only factor capable of securing macrostability.

The various mental and social microprocesses that together achieve cultural propagation combine preservative and constructive functions in different degrees. (We talk of "preservative" rather than "reproductive" or "replicative" function, because, unlike genes, cultural material does not selfreproduce; rather, it serves as input to intra- and interindividual processes that tend to preserve their informational content in their output.) If, instead of postulating that the preservative function of memory, imitation, and communication must be effective enough to explain cultural macrostability, one pays attention to the vast psychological and sociological literature on these processes, then it becomes clear that high fidelity is the exception rather than the rule.

\section{Constructive Propagation}

Outputs of individual memory, imitation, and communication processes are, quite generally, not copies but transformations of the inputs. This is due in part to the imperfection of these mechanisms: some information is just lost in the process of transmission. It is also due to the very function of these mechanisms, which is never purely preservative. Even when they are faithful, the microprocesses of cultural propagation are in good part constructive rather than replicative (Sperber 1996, 2000). The preservation of information is a means toward the end of providing individuals with information adapted to their mental and motor schemas and to the pursuit of their goals. The output of the mechanisms involved is only in part based on the input information to be remembered or transmitted; it also draws on general and contextual information and is shaped by the constructive functions of these mechanisms.

The fact that processes of cultural propagation are partly preservative and partly constructive affects the ways in which cultural stability itself can be explained. If the processes involved were just preservative, an occasional error of replication (akin to a mutation) would be preserved in further errorless replications: it would become the model. If such copying errors were frequent, heritability might be too low_or, equivalently, the mutation rate might be too high-for selection to be effective, or for anything to propagate at all. Given that the processes involved in cultural propagation are partly constructive, failures to faithfully reproduce the inputs of these processes are not, properly speaking, "copy errors." Rather than resulting in random drift, these constructive transformations may be biased toward producing outcomes that are more suited to the individual's cognitive and practical abilities and goals.

Here is how the partly constructive character of the processes involved in cultural propagation helps explain cultural stability (alongside other mechanisms such as the decision biases described by Richerson and Boyd 2005; see Sperber and Claidière in press for discussion). Many constructive biases are shared in a population. This may be due to the fact that they are based on the common psychological makeup of the species, or to more local historical or ecological factors. Shared biases may not only statistically compensate the low fidelity of preservative microprocesses; they may also permit some items to reach a cultural level of distribution and stability.

Here is a simple example. The region of the continuum of colors referred to by a given color term, say "red," does not have clear boundaries, but it has, for every user, a focal point which is seen as prototypical red. In learning the meaning of "red," a child is not taking the first sample he/she hears described as "red" as prototypical red, nor is he/she averaging over all the samples that he/she hears described as "red" in his/her learning period. Rather, the child's color perception system provides him/her with a ready-made focal point for red on the basis of which he/she interprets the word. The child may depart from the samples of "red" he/she is presented with, in the direction of what is a more salient identification, given his/her perceptual dispositions. Because the color focal points for red and other basic colors are similar across individuals, they stabilize rough common meanings for basic color terms in any given language, and terms with the same focal points across many languages, in spite of the fact that interlocutors hardly ever mean exactly the same thing by "red" (Berlin and Kay 1969; Kay et al. 1997).

\section{Cultural Causality Is Promiscuous}

We can now spell out the most profound disanalogy between biological and cultural evolution. Among the causal factors determining biological evolution, there is sharp distinction between, on the one hand, an extraordinarily robust mechanism of inheritance-replication - without which there would be no biological evolution at all, and, on the other hand, a variety of environmental factors (physical factors, inter- and intraspecies factors, intergene factors, cytoplasmic environment, and so on) that affect evolution by determining the relative success 
of different genes. The factors determining cultural evolution cannot be similarly divided into replication mechanisms and environmental factors. While some mechanisms (of memory, imitation, and communication) have a primarily preservative function, they themselves involve constructive processes drawing extensively on contextual information delivered by other mechanisms (for instance mental mechanisms of perception, inference, or action planning, and social processes of seduction, coercion, deception, bargaining, coalition formation, and so on), the primary function of which is not preservative. Constructive processes systematically intrude on preservative processes. Cultural contents are not replicated by one set of inheritance mechanisms and selected by another, disjoint set of environmental factors-not by a long shot.

We are well aware of the fact that biological transmission is much more complex than a few sentences can indicate, and that it involves a variety of submechanisms that may have analogues in cultural evolution. To give just one illustration, consider an orally transmitted folktale. The public telling of the tale contributes to the formation in the listeners of mental representations of the tale, and these mental representations contribute to the production of further public telling by listeners turned tellers. The causal chain involves an alternation of mental and public events with equally potent causal roles. The case of RNA retroviruses that propagate through reverse transcription into DNA might be seen as a biological analogue. But how helpful would such an analogy be?

Merely adjusting the general model of Darwinian selection to describe cultural evolution involves idealizing away properties crucial to the proper explanation of the phenomena. Looking to biology for fine-grained analogies between biological and cultural evolution may be a source of insights but it should not take precedence over looking to the cognitive and social sciences for a better understanding of the actual mechanisms and processes involved. We agree with Richerson and Boyd (2005) that Darwin-inspired population thinking provides the proper approach to the scientific study of cultural evolution. However, it has yet to be generalized to cases where the causal forces involved cannot be neatly separated into reproductive mechanisms and environmental factors. Cultural causality is promiscuous and that is why modeling it is still such a challenge.

\section{References}

Aunger R (2002) The Electric Meme: A New Theory of How We Think. New York: Free Press.

Berlin B, Kay P (1969) Basic Color Terms: Their Universality and Evolution. Berkeley: University of California Press.

Bloom P (2000) How Children Learn the Meanings of Words. Cambridge, MA: MIT Press.

Boyd R, Richerson PJ (1985) Culture and the Evolutionary Process. Chicago: University of Chicago Press.

Campbell DT (1960) Blind variation and selective retention in creative thought as in other knowledge processes. Psychological Review 67: 380-400.

Cavalli-Sforza LL, Feldman MW (1981) Cultural Transmission and Evolution. Princeton: Princeton University Press.

Cohen L, Dehaene S (2004) Specialization within the ventral stream: The case for the visual word form area. NeuroImage 22: 466-476.

Dawkins R (1976) The Selfish Gene. Oxford: Oxford University Press.

Durham W (1991) Coevolution: Genes, Culture, and Human Diversity. Stanford: Stanford University Press.

Kay P, Berlin B, Maffi L, Merrifield W (1997) Color naming across languages. In: Color Categories in Thought and Language (Hardin CL, Maffi L, eds), 21-58. Cambridge: Cambridge University Press.

Mazoyer BM, Tzourio N, Frak V, Syrota A, Murayama N, Levrier O, Salamon G, Dehaene S, Cohen L, Mehler J (1993) The cortical representation of speech. Journal of Cognitive Neuroscience 5: 467-479.

Mesoudi A, Whiten A, Laland K (2004) Is human cultural evolution Darwinian? Evidence reviewed from the perspective of The origin of species. Evolution 58: 1-11.

Monod J (1970) Le hasard et la nécessité. Paris: Seuil.

Richerson PJ, Boyd R (2005) Not by Genes Alone. Chicago: University of Chicago Press.

Sperber D (1996) Explaining Culture: A Naturalistic Approach. Oxford: Blackwell.

Sperber D (2000) An objection to the memetic approach to culture. In: Darwinizing Culture (Aunger R, ed), 163-174. Oxford: Oxford University Press.

Sperber D, Claidière N. (in press) Defining and explaining culture (comments on Richerson and Boyd, Not by genes alone). Biology and Philosophy.

Sperber D, Hirschfeld L (1999) Evolution, cognition and culture. In: MIT Encyclopedia of the Cognitive Sciences (Keil F, Wilson R, eds), cxicxxxii. Cambridge, MA: MIT Press. 
This article has been cited by:

1. Christophe Heintz. 2007. Institutions as Mechanisms of Cultural Evolution: Prospects of the Epidemiological Approach. Biological Theory 2:3, 244-249. [Abstract] [PDF] [PDF Plus]

2. Alex Mesoudi. 2007. Biological and Cultural Evolution: Similar but Different. Biological Theory 2:2, 119-123. [Citation] [PDF] [PDF Plus] 